\title{
Polymer Molded Templates for Nanostructured Amorphous Silicon Photovoltaics
}

Lei Pei

Amy Balls

Cary Tippets

Jonathan Abbott

Matthew R. Linford

Follow this and additional works at: https://scholarsarchive.byu.edu/facpub See next page for additional authors

Part of the Astrophysics and Astronomy Commons, and the Physics Commons

\section{Original Publication Citation}

The following article appeared in Lei Pei*, Amy Balls, Cary Tippets, Jonathan Abbott*, Matthew Linford, Jian Hu*, Arun Madan, David Allred, Richard Vanfleet, and Robert Davis, "Polymer Molded Templates for Nanostructured Amorphous Silicon Photovoltaics," Journal of Vacuum Science \& Technology A 29(2), (211). and can be found at [http://avspublications.org/jvsta/ resource/1/jvtad6/v29/i2/p2117_s1][http://dx.doi.org/1.1116/1.355472].'

\section{BYU ScholarsArchive Citation}

Pei, Lei; Balls, Amy; Tippets, Cary; Abbott, Jonathan; Linford, Matthew R.; Allred, David D.; Vanfleet, Richard R.; Davis, Robert C.; Hu, Jian; and Madan, Arun, "Polymer Molded Templates for Nanostructured Amorphous Silicon Photovoltaics" (2011). Faculty Publications. 817.

https://scholarsarchive.byu.edu/facpub/817

This Peer-Reviewed Article is brought to you for free and open access by BYU ScholarsArchive. It has been accepted for inclusion in Faculty Publications by an authorized administrator of BYU ScholarsArchive. For more information, please contact ellen_amatangelo@byu.edu. 


\section{Authors}

Lei Pei, Amy Balls, Cary Tippets, Jonathan Abbott, Matthew R. Linford, David D. Allred, Richard R. Vanfleet, Robert C. Davis, Jian Hu, and Arun Madan 


\title{
Polymer molded templates for nanostructured amorphous silicon photovoltaics $^{\text {a) }}$
}

\author{
Lei Pei \\ Department of Chemistry and Biochemistry, Brigham Young University, Provo, Utah 84602 \\ Amy Balls, Cary Tippets, and Jonathan Abbott \\ Department of Physics and Astronomy, Brigham Young University, Provo, Utah 84602 \\ Matthew R. Linford \\ Department of Chemistry and Biochemistry, Brigham Young University, Provo, Utah 84602 \\ Jian Hu and Arun Madan \\ MVSystems, Inc., Golden, Colorado 80401 \\ David D. Allred, Richard R. Vanfleet, and Robert C. Davis ${ }^{\text {b) }}$ \\ Department of Physics and Astronomy, Brigham Young University, Provo, Utah 84602 \\ (Received 22 November 2010; accepted 20 January 2011; published 22 February 2011)

\begin{abstract}
Here, the authors report the fabrication of transparent polymer templates for nanostructured amorphous silicon photovoltaics using low-cost nanoimprint lithography of polydimethylsiloxane. The template contains a square two-dimensional array of high-aspect-ratio nanoholes $(300 \mathrm{~nm}$ diameter by $1 \mu \mathrm{m}$ deep holes) on a $500 \times 500 \mathrm{~nm}^{2}$ pitch. A $100 \mathrm{~nm}$ thick layer of $a-\mathrm{Si}: \mathrm{H}$ was deposited on the template surface resulting in a periodically nanostructured film. The optical characterization of the nanopatterned film showed lower light transmission at $600-850 \mathrm{~nm}$ wavelengths and lower light reflection at 400-650 nm wavelengths, resulting in $20 \%$ higher optical absorbance at AM 1.5 spectral irradiance versus a nonpatterned film. (C) 2011 American Vacuum Society. [DOI: 10.1116/1.3554720]
\end{abstract}

\section{INTRODUCTION}

Compared to crystalline silicon based solar cells, thin-film hydrogenated amorphous silicon $(a-\mathrm{Si}: \mathrm{H})$ solar cells have the potential advantages of less raw material usage and lower fabrication costs as well as the benefits of high flexibility and light weight. However, the efficiency of single-layer thinfilm $a$-Si:H solar cells is relatively low. Several efforts have been made to increase single-layer cell efficiency by improving the light trapping of devices.

To achieve efficient light trapping researchers have developed a number of scattering techniques. These include randomly textured substrates, such as Asahi-U type glass ${ }^{1}$ and other high-haze transparent conductive oxide material coatings $^{2}$ which scatter light at their rough interfaces. The scattered light increases the effective optical path length for internally diffused light rays, which widens the absorption window toward long light wavelengths. Metal nanoparticles $^{3,4}$ have also been incorporated into photovoltaic systems as plasmonic structures for light scattering and for exciting near-field charge carriers and coupling light into guided modes. These techniques have been applied either on the front or rear of the solar cell. ${ }^{5,6}$ Periodically nanostructured plasmonic back contacts have been reported to improve short-circuit current densities in the spectral range from 550 to $800 \mathrm{~nm}$. These demonstrated light trapping, enhanced beyond that of a randomly textured cell. ${ }^{7}$ Another surface-

\footnotetext{
a) This paper was presented at the 57th Symposium of the American Vacuum Society, Albuquerque, MN, October 20, 2010.

${ }^{b}$ Electronic mail: davis@byu.edu
}

texture geometry that has resulted in increased efficiencies is the nanodome solar cell device with significantly higher absorption than a flat solar cell. ${ }^{8}$ Subwavelength gratings, which have consisted of a one-dimensional array of lines with pitch smaller than the light wavelength, have been used to suppress reflection over wide spectral bandwidth and large field of view. ${ }^{9,10}$ In photovoltaic applications, ${ }^{3,11-15}$ for example, periodically structured $\mathrm{ZnO}: \mathrm{Al}$ front contacts ${ }^{12}$ have been implemented in amorphous silicon thin-film solar cells to reduce reflectance and increase current density.

To directly measure light absorption in periodic nanopatterned $a$-Si:H films, we designed and fabricated a polymer template with a square two-dimensional array of high-aspectratio nanoholes ( $300 \mathrm{~nm}$ diameter by $1 \mu \mathrm{m}$ deep holes) on a $500 \times 500 \mathrm{~nm}^{2}$ pitch. A $100 \mathrm{~nm}$ thick layer of $a$-Si:H was deposited on the template surface resulting in a periodically nanostructured film and on planar surfaces for comparison. Optical reflection and transmission measurements were then conducted with an integrating sphere to capture both specular and scattered lights. In order to measure the effect of nanoscale structuring on absorption of the amorphous silicon without absorption contributions by other adjacent materials, a simplified sample was fabricated without any metal back reflector or electrode coating.

Besides the analysis of optical absorption, several other aspects have been considered in this nanostructure design: (1) the template can be cost-effectively incorporated into a single junction thin-film photovoltaic (PV) device using conventional PV film deposition techniques; (2) fast scalable production of the template using roll-to-roll techniques ${ }^{16}$ can 


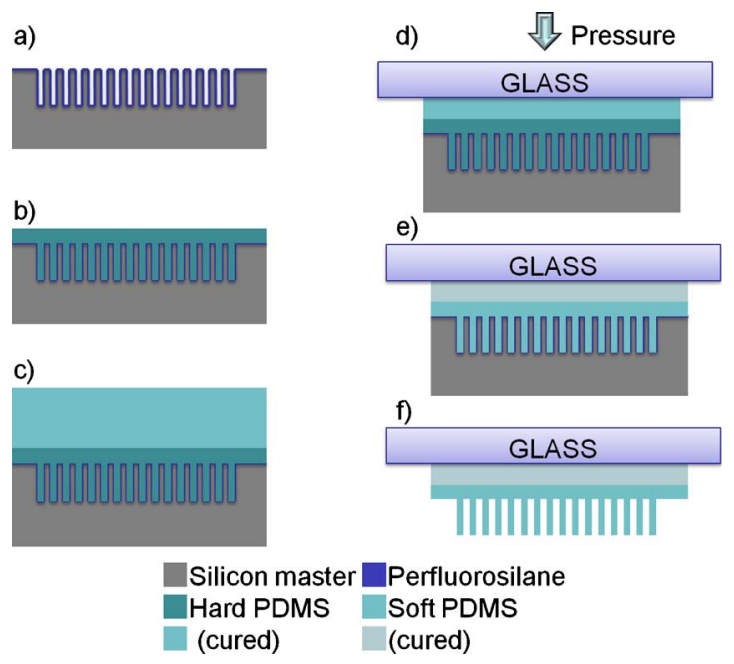

FIG. 1. (Color online) Scheme of nanoimprint process: (a) silicon master coated with perfluorosilane; (b) spin coating hard PDMS; (c) pouring soft PDMS on top of the hard PDMS; (d) placing a glass slide; (e) curing the PDMS stack; and (f) releasing the silicon master.

be applied on inexpensive polymer substrates; and (3) the patterned photovoltaic approach, when implemented at the appropriate dimensions, could have an impact on using PV materials characterized by high defect densities including organic, quantum dot, and oxide PV systems.

\section{EXPERIMENT}

The fabrication process for our three-dimensional nanostructured polymer template has two main steps: silicon master fabrication and nanoembossing. A reusable silicon master was first produced with a nanopillar array. This silicon master was used as a mold to emboss on a polymer film via nanoimprint lithography. This is a technique for high throughput patterning of polymer nanostructures at great precision and at low costs. ${ }^{17,18}$ The process diagram is shown in Fig. 1.

\section{A. Electron-beam lithography}

ZEP 520A e-beam resist (Nippon Zeon) was spin coated on a silicon wafer and baked on a hotplate at $170{ }^{\circ} \mathrm{C}$ for 2 min. ZEP has high sensitivity which is particularly valuable for large area patterning and has relatively high plasma etch resistance. To reduce resist charging during exposure, a layer of conductive polymer was also spin coated on top of the ZEP layer and baked on a hotplate at $90{ }^{\circ} \mathrm{C}$ for $30 \mathrm{~s}$. The designed pattern was a square about $5 \times 5 \mathrm{~mm}^{2}$. This pattern contains an $18 \times 18$ array of $300 \times 300 \mu \mathrm{m}^{2}$ squares with a $5 \mu \mathrm{m}$ overlap of the squares on each side. The $300 \mu \mathrm{m}$ squares are made of a series of perpendicularly crossed lines with $500 \mathrm{~nm}$ pitch to form a grid pattern. An FEI/Philips XL30 FEG ESEM (operating in high vacuum mode) with a nanometer pattern generation system (JC Nabity Lithography Systems) was used for electron beam exposure. The electron beam was set at $30 \mathrm{kV}$ and the spot was set to size 5 (1172 pA) to allow fast writing. The magnification was $200 \times$ and

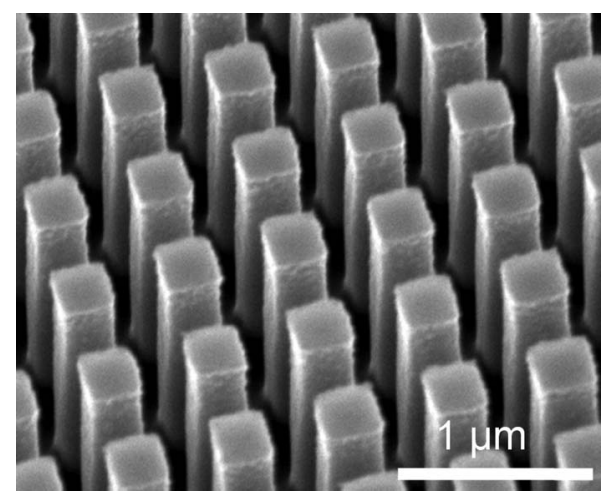

FIG. 2. SEM image of the patterned silicon wafer with nanopillar array.

the exposure line dose was $0.867 \mathrm{nC} / \mathrm{cm}$. The exposed ZEP sample was first rinsed with deionized (DI) water to remove the conductive polymer, followed by development in ZEDN50 developer, rinsing in ZMD-D, and again rinsing in DI water. Finally, the sample was put into an oxygen plasma etcher (Technics Planar-Etch II) at $100 \mathrm{~W}$ for $1 \mathrm{~min}$ to descum the resist pattern.

\section{B. Silicon master etching}

The sample was then loaded into a multiplex inductively coupled plasma reactive ion etcher (Surface Technology Systems) with $\mathrm{SF}_{6}$ and $\mathrm{C}_{4} \mathrm{~F}_{8}$ flow for directional etching. The exposed regions of the silicon surface were etched down $1 \mu \mathrm{m}$. After etching, the sample was put into the PE2 oxygen plasma etcher to remove most of the e-beam resist. The sample was then treated in piranha solution to thoroughly clean and to add hydroxyl groups on the surface.

\section{Antiadhesion coating deposition}

A perfluorosilane antiadhesion layer was deposited on the surface of the silicon master via following steps. The silicon master was put into a desiccator. A drop or two of trichloro $(1 \mathrm{H}, 1 \mathrm{H}, 2 \mathrm{H}, 2 \mathrm{H}$-perfluorooctyl)silane (448931, Sigma-Aldrich) were deposited in two scintillation vials which were placed beside the master. The desiccator chamber was pumped down with the laboratory vacuum for 10 min to accelerate the silane vaporization. Finally, the desiccator was sealed overnight to allow sufficient deposition on the high-aspect-ratio pattern. This perfluorosilane antiadhesion coating had an advancing water contact angle over $118^{\circ}$, which helps the silicon master separate from imprinted polymer at the end of the nanoimprint process. Figure 2 shows the resulting silicon nanopillar array master.

\section{Nanoimprint patterning of the polymer}

Polydimethylsiloxane (PDMS) was used for the patterned polymer template. PDMS has the advantages of (1) low cost, (2) optical transparency, and (3) thermal stability (well over the temperature of $200{ }^{\circ} \mathrm{C}$ required for the silicon layer deposition). Both hard PDMS and soft PDMS were employed in the process. The hard PDMS has a higher modulus 
of elasticity and is used to replicate the high-resolution highaspect-ratio template features. Soft PDMS was used as a glue to attach the hard PDMS to a glass slide.

To prepare hard PDMS, $3.4 \mathrm{~g}$ of a vinyl PDMS prepolymer (VDT-731, Gelest Corp.), $18 \mu \mathrm{L}$ of a Pt catalyst (platinum divinyltetramethyldisiloxane, SIP6831.1, Gelest Corp.), and one drop of a modulator (2,4,6,8-tetramethyltetravinylcyclotetrasiloxane, 87927, Sigma-Aldrich) were mixed and degassed for $15 \mathrm{~min}$ in a vacuum desiccator. $1 \mathrm{~g}$ of a hydrosilane prepolymer (HMS-301, Gelest Corp.) was then gently stirred into this mixture and degassed for another 2 min. Immediately (within $3 \mathrm{~min}$ ), this hard PDMS mixture was spin coated onto the silicon master at $1000 \mathrm{rpm}$ for $40 \mathrm{~s}$, forming a $30-40 \mu \mathrm{m}$ thick layer. Hard PDMS thus fully covered the nanopillars on the silicon master. The coated master was then put into an oven and partially cured for 30 $\min$ at $60{ }^{\circ} \mathrm{C}$.

The soft PDMS prepolymer Sylgard 184 PDMS (Dow Corning) base and curing agent were mixed 10:1 and degassed for $30 \mathrm{~min}$ ahead of time. This liquid prepolymer was poured onto the hard PDMS layer immediately after the partial curing, while the hard PDMS was still slightly tacky. The partially completed structure was then degassed in a vacuum desiccator for $1 \mathrm{~h}$. After a glass slide was placed on the soft PDMS and a $530 \mathrm{~g}$ weight was placed on the glass slide (Fig. $1)$, the structure was returned to the vacuum for further degassing to remove any bubbles between the soft PDMS and the glass. The PDMS stack was slowly cured at room temperature (overnight, 12-14 h). Fast curing at high temperature can cause the hard PDMS to crack. After this slow curing, the entire assembly was placed in an oven at $60{ }^{\circ} \mathrm{C}$ for a $1 \mathrm{~h}$ hard bake. The composite PDMS template was then released from the master surface by carefully peeling the template from the surface while they were still warm. Figure 3 shows the optical and scanning electron microscopy (SEM) images of the imprinted nanoholes, which are the inverse of the silicon master pattern.

\section{E. Photovoltaic material deposition}

Patterned and unpatterned PDMS layer regions on a glass slide were simultaneously coated with $100 \mathrm{~nm}$ amorphous silicon by plasma enhanced chemical vapor deposition (PECVD). The deposition was done in an MVSystems PECVD reactor (MVSystems, Inc.) at $200{ }^{\circ} \mathrm{C}$ from pure silane gas. The amorphous silicon produced in this study results in a typical hydrogen concentration of $\sim 10 \%$; the optical bandgap is of 1.7-1.8 eV. For planar amorphous silicon layers of $100 \mathrm{~nm}$ thickness, the largest gain in absorption is in the wavelength range of $400-540 \mathrm{~nm}$ as the absorption coefficient of the material becomes $>1 \times 10^{5} / \mathrm{cm}$ in this wavelength range. For sample description, the area outside the nanostructured region is labeled "planar" sample, while the area inside nanostructured template is labeled as a "patterned" sample; both samples consisted of a glass/PDMS/a-Si:H stack. The $100 \mathrm{~nm}$ layer thickness was chosen because in amorphous silicon devices the StaeblerWronski degradation limits the active layer thickness for
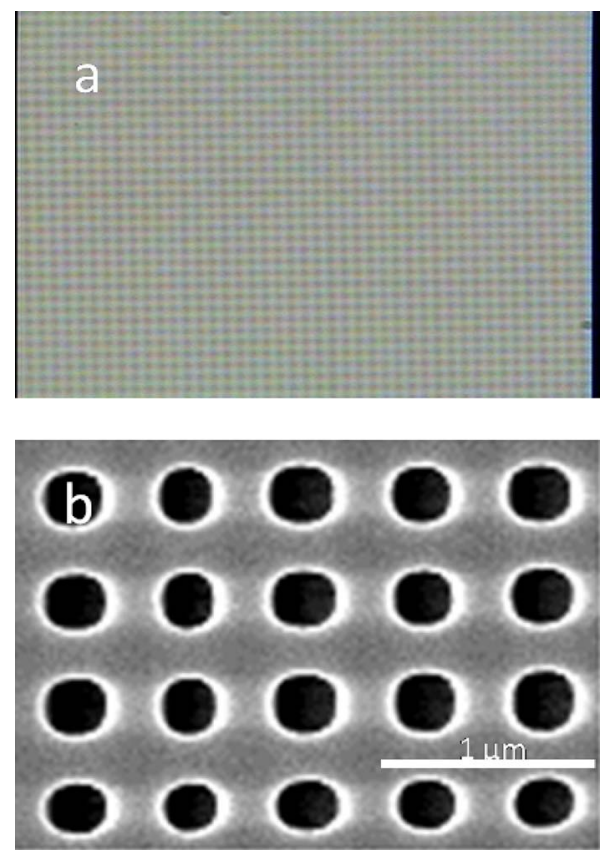

FIG. 3. (Color online) (a) Optical and (b) SEM images of embossed nanoholes.

highly efficient carrier extraction to approximately $100 \mathrm{~nm} .{ }^{19}$ At this thickness, however, planar layers capture little of the light at wavelengths above $600 \mathrm{~nm}$. Figure 4(a) shows a SEM front view of the patterned sample. The diameter of the nanoholes was reduced by around $100 \mathrm{~nm}$ on the surface by the amorphous silicon deposition. In the cross-section image [Fig. 4(b)] processed by focused ion beam (FIB), $100 \mathrm{~nm}$ of amorphous silicon (which shows up as black) can be seen deposited mostly at the top of the posts with the coating continuing down the posts approximately $150 \mathrm{~nm}$.

\section{F. Optical measurement setup}

Reflectance and transmission as a function of wavelength were measured on both patterned and planar samples. A tungsten lamp was used as the light source. The light beam was collimated with a lens tube. Then the light passed through an aperture to reduce the beam diameter to approximately $3 \mathrm{~mm}$, which is small enough to easily align with the $5 \times 5 \mathrm{~mm}^{2}$ nanopatterned area. A 2 in. diameter integrating sphere with a 99\% reflectance coating (Thorlabs, IS236A-4)
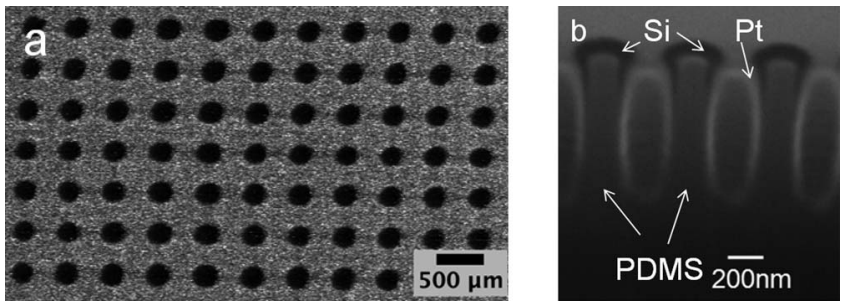

FIG. 4. (a) SEM top view of $a$-Si coated patterned polymer template; (b) cross-section view presents Pt (FIB deposited), $a$-Si (dark cap), and PDMS post, starting from the top and moving down a post. 

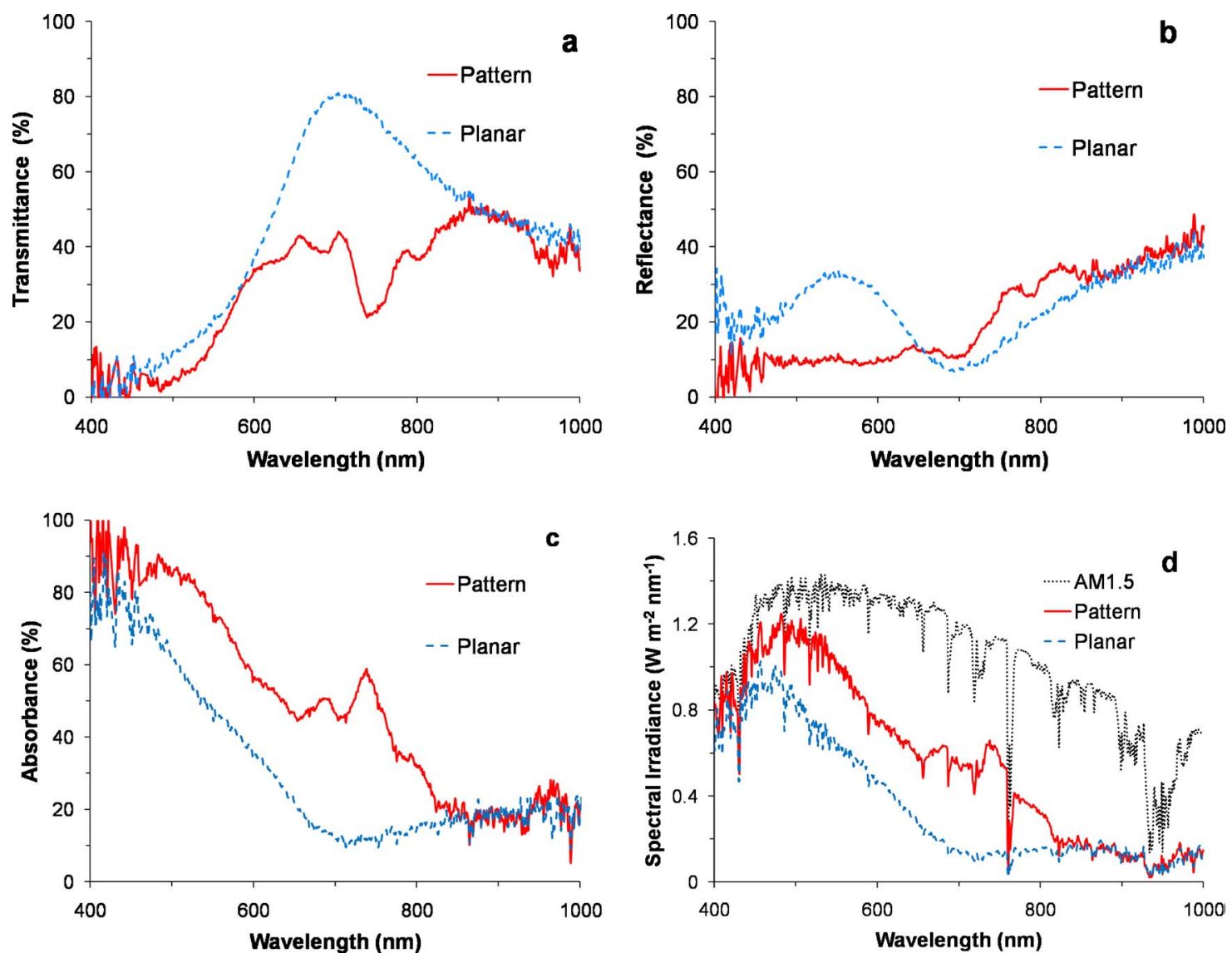

FIG. 5. (Color online) (a) Transmittance spectra of patterned and planar areas; (b) reflectance spectra of patterned and planar areas; (c) absorbance spectra of patterned and planer areas; and (d) spectral irradiance of AM 1.5 and weighted absorbance of patterned and planer areas.

was used for transmission, reflection, and incident beam intensity measurements. A Czerny-Turner charge-coupled device (CCD) spectrometer (Science-Surplus, compact fiber coupled CCD spectrometer, 365-1100 nm) was fiber coupled to the detector port of the integrating sphere.

For the total beam intensity measurements, the entrance port of the integrating sphere was opened but the sample port at $180^{\circ}$ was closed. The incident beam passed through the center of the entrance port striking the diffuse white reflective surface on the sample port scattering evenly around the sphere. The obtained spectrum was used as the "total" beam intensity to compare with the transmission and reflection spectra. For transmission measurements the sample was placed perpendicular to the incident beam in front of the entrance port. For reflection measurements the $180^{\circ}$ port of the integrating sphere (opposite the entrance port) was opened and the sample, tilted at a $5^{\circ}$ angle relative to the perpendicular, was placed up against this port. The tilt on the sample insures that light reflected specularly from the sample hits the side of the integrating sphere. Without it specular light could leave through the entrance port and not reach the detector.

\section{OPTICAL RESULTS AND DISCUSSION}

The transmittance (relative to total beam) spectra [Fig. 5(a)] show a significant difference between patterned and planar samples at longer wavelengths $(600-850 \mathrm{~nm})$. Especially around $700-750 \mathrm{~nm}$, the patterned sample transmits $40 \%-50 \%$ less light than the planar sample. Lower light reflection of the patterned sample was also observed at 400$650 \mathrm{~nm}$ wavelengths [Fig. 5(b)]. There are many sharper optical features seen between 600 and $800 \mathrm{~nm}$; we attribute these features to complex optical resonances of our subwavelength periodic structures.

The absorbance spectra [Fig. 5(c)] were calculated from transmittance and reflectance spectra. The spectra show that the patterned area has higher absorption in most of the visible spectrum. At longer wavelengths $(650-800 \mathrm{~nm})$, where the absorption of the planar area is very low, the patterned area has significantly more absorption.

The weighted absorbance spectra [Fig. 5(d)] were calculated from the absorbance spectra weighted by the spectral irradiance AM 1.5 spectra. The integrated weighted absorbance for patterned and planar samples shows that the patterned sample captured 55\% of spectral irradiance energy, while the planar sample only got $35 \%$. Moreover, this $20 \%$ improvement might be further increased by optimizing the amorphous silicon deposition profile.

Experimentally it is possible that in transmission (reflection) measurements light scattered (reflected) at wide angles $\left(>70^{\circ}\right.$ relative to the normal) will not enter into and be collected in the integrating sphere due to a $\pm 70^{\circ}$ acceptance 
angle of the sphere ports. Such light could appear as an increase in absorption. Also, there is drift in the light source as well as detector noise that will result in some uncertainty in the absorption measurement. By measuring reflection and transmission on patterned and unpatterned transparent PDMS only samples, we were able to put an upper bound on the uncertainty of the absorption measurement due to systematic factors such as noncollected light. There is some wide-angle low-intensity scattering $\left(>70^{\circ}\right.$ from normal) from the patterned PDMS sample; however, reflection and transmission measurements account for all of the incident light with less than $5 \%$ uncertainty. There is similar wideangle scattering from the $a$-Si:H coated template but it is much dimmer than from the transparent PDMS sample; it should therefore result in measurement uncertainties less than $5 \%$ of incident.

The increased absorption at longer wavelengths is not due to lower reflection; that is, the effect is not simply antireflection. In fact, at longer wavelengths (600-800 nm) the reflection of patterned samples is slightly higher than the reflection of planar samples. Nevertheless, at these long wavelengths the patterned sample absorption is much higher. Since the only absorbing material in the sample is amorphous silicon, this difference results from increased absorption in the silicon. Since there is the same amount of silicon per area as the planar sample, the effective light path through the silicon must be longer. This is consistent with coupling of the light into in-plane guided modes as proposed by other researchers at grating periods on this scale. ${ }^{7,14}$ However, in prior work, direct measurement of absorption by the semiconductor has not been possible due to the presence of nanopatterned metal layers. Our results here indicate that the nanopatterned semiconductor itself acts as an effective coupling grating.

Periodic patterning can also minimize reflection, as we see at the short wavelength end of the spectrum in Fig. 5(b). Eisele et al. ${ }^{12}$ showed that periodic patterning reduced reflections most effectively at $980 \mathrm{~nm}$ pitch. However, a shorter pitch is likely required for maximum coupling of the light into guided modes. Our absorption work indicates that with a $500 \mathrm{~nm}$ pitch we are effectively coupling 600-800 nm light, increasing absorption at the wavelengths most critical for light capture in amorphous silicon. Light capture is critical at the long wavelength end of the visible spectrum because $a-\mathrm{Si}$ has such a very small absorption coefficient there. This is also in contrast to random textures that increase absorption nonresonantly resulting in a smaller absorption gain where it is most needed. ${ }^{7}$

\section{CONCLUSION}

We have developed a low-cost-template fabrication process for nanostructuring of amorphous silicon into a layer with a two-dimensional array of holes. This geometry was a convenient platform for optical absorption measurements on nanopatterned semiconductor layers. The increased absorption in the nanohole array indicates coupling to lateral guided surface modes. This approach provides a test bed for absorption studies in nanostructured film geometries and should result in improved light capturing designs in thin-film solar cells.

\section{ACKNOWLEDGMENTS}

The authors acknowledge the support of CHIRP Grant from the College of Physical and Mathematical Sciences at Brigham Young University. One of the authors (C.T.) was supported by a grant from the BYU Office of Research and Creative Activities. The authors thank Steven Schultz of the BYU Department of Electrical Engineering for useful discussions.

${ }^{1}$ K. Sato, Y. Gotoh, Y. Wakayama, Y. Hayasahi, K. Adachi, and H. Nishimura, Rep. Res. Lab. Asahi Glass Co. Ltd. 42, 129 (1992).

${ }^{2}$ J. Krč, F. Smole, and M. Topič, Prog. Photovoltaics 11, 429 (2003).

${ }^{3}$ H. A. Atwater and A. Polman, Nature Mater. 9, 205 (2010).

${ }^{4}$ K. Nakayama, K. Tanabe, and H. A. Atwater, Appl. Phys. Lett. 93, 121904 (2008).

${ }^{5}$ R. H. Franken, R. L. Stolk, H. Li, C. H. M. van der Werf, J. K. Rath, and R. E. I. Schropp, J. Appl. Phys. 102, 014503 (2007).

${ }^{6}$ S. Fahr, C. Rockstuhl, and F. Lederer, Appl. Phys. Lett. 92, 171114 (2008).

${ }^{7}$ V. E. Ferry, M. A. Verschuuren, H. B. T. Li, E. Verhagen, R. J. Walters, R. E. I. Schropp, H. A. Atwater, and A. Polman, Opt. Express 18, A237 (2010).

${ }^{8}$ J. Zhu, C. M. Hsu, Z. F. Yu, S. H. Fan, and Y. Cui, Nano Lett. 10, 1979 (2010).

${ }^{9}$ Y. Kanamori, E. Roy, and Y. Chen, Microelectron. Eng. 78-79, 287 (2005).

${ }^{10}$ Y. Kanamori, K. Kobayashi, H. Yugami, and K. Hane, Jpn. J. Appl. Phys., Part 1 42, 4020 (2003).

${ }^{11}$ N. Senoussaoui, M. Krause, J. Muller, E. Bunte, I. Brammer, and H. Stiebig, Thin Solid Films 451-452, 397 (2004).

${ }^{12}$ C. Eisele, C. E. Nebel, and M. Stutzmann, J. Appl. Phys. 89, 7722 (2001).

${ }^{13}$ K. Sopian, N. Asim, N. Amin, and S. H. Zaidi, European Journal of Scientific Research 24, 358 (2008).

${ }^{14}$ K. Söderström, F. J. Haug, J. Escarré, O. Cubero, and C. Ballif, Appl. Phys. Lett. 96, 213508 (2010).

${ }^{15}$ C. Haase and H. Stiebig, Prog. Photovoltaics 14, 629 (2006).

${ }^{16}$ R. E. I. Schropp and M. Zeman, IEEE Trans. Electron Devices 46, 2086 (1999).

${ }^{17}$ L. J. Guo, Adv. Mater. (Weinheim, Ger.) 19, 495 (2007).

${ }^{18}$ T. W. Odom, J. C. Love, D. B. Wolfe, K. E. Paul, and G. M. Whitesides, Langmuir 18, 5314 (2002).

${ }^{19}$ A. Luque and S. Hegedus, Knovel (Firm), Handbook of Photovoltaic Science and Engineering (Wiley, Hoboken, NJ, 2003), p. 1138. 